\title{
Modelling pertussis transmission to evaluate the effectiveness of an adolescent booster in Argentina
}

\author{
G. FABRICIUS ${ }^{1 *}$, P. E. BERGERO ${ }^{1}$, M. E. ORMAZABAL ${ }^{2}$, A. L. MALTZ ${ }^{3}$ \\ AND D. F. HOZBOR ${ }^{2}$ \\ ${ }^{1}$ Instituto de Investigaciones Fisicoquímicas Teóricas y Aplicadas, Facultad de Ciencias Exactas, \\ Universidad Nacional de La Plata, La Plata, Argentina \\ ${ }^{2}$ Laboratorio VacSal, Instituto de Biotecnología y Biología Molecular, Departamento de Ciencias Biológicas, \\ Facultad de Ciencias Exactas, Universidad Nacional de La Plata, CCT-La Plata, CONICET, La Plata, \\ Argentina \\ ${ }^{3}$ Departamento de Matemática, Universidad Nacional de La Plata, La Plata, Argentina
}

Received 8 April 2012; Final revision 18 May 2012; Accepted 31 May 2012; first published online 6 July 2012

\section{SUMMARY}

Due to the current epidemiological situation of pertussis, several countries have implemented vaccination strategies that include a booster dose for adolescents. Since there is still no evidence showing that the adolescent booster has a positive effect on the most vulnerable group represented by infants, it is difficult to universalize the recommendation to include such reinforcement. In this work we present an age-structured compartmental deterministic model that considers the outstanding epidemiological features of the disease in order to assess the impact of the booster dose at age 11 years (Tdap booster) to infants. To this end, we performed different parameterizations of the model that represent distinct possible epidemiological scenarios. The results obtained show that the inclusion of a single Tdap dose at age 11 years significantly reduces the incidence of the disease within this age group, but has a very low impact on the risk group $(0-1$ year). An effort to improve the coverage of the first dose would have a much greater impact on infants. These results hold in the 18 scenarios considered, which demonstrates the robustness of these conclusions.

Key words: Epidemic, mathematical model, pertussis, Tdap booster.

\section{INTRODUCTION}

Bordetella pertussis causes the respiratory disease named whooping cough or pertussis that is most severe in infants. Before massive vaccination was introduced in the 1950s, pertussis was a major cause of infant mortality worldwide. As a consequence of

\footnotetext{
* Author for correspondence: Dr G. Fabricius, Instituto de Investigaciones Fisicoquímicas Teóricas y Aplicadas, Diagonal 113 y 64, Cc. 16, Suc. 4, 1900 La Plata, Argentina.

(Email: fabricius@fisica.unlp.edu.ar)
}

the implementation of a pertussis immunization programme, a marked decline in disease incidence was observed [1, 2]. However, in the 1990s a resurgence of pertussis was detected in different countries and now pertussis has become the most prevalent vaccinepreventable disease in developed and in developing countries [3-5]. The disease is now recognized as a frequent infection not only for infants but also for adolescent and adults [6]. Several explanations have been proposed for this resurgence of the disease, including improved surveillance, waning immunity 
and bacterial evolution [5, 7-10]. The relevance of these factors in the resurgence of pertussis might differ between countries. Beyond the possible causes, it is clear that the number of cases has steadily increased which undoubtedly marks the need to revise and implement strategies to improve the disease control. In fact, in 2002 different ministries of health, committees and organizations like the Global Pertussis Initiative (GPI, 2002), and Advisory Committee on Immunization Practices (ACIP) of the USA recommended expanding the existing vaccination strategies to include a pertussis booster dose for adolescents [11, 12]. The reported rationale for that strategy is to provide both direct protection for the adolescents that receive the booster and indirect benefit for non-immunized peers and the most vulnerable age groups, the infants. For this secondary but important goal, however, there is still no evidence showing a reduction in severe infant disease and mortality even following the implementation of an adolescent booster dose at age 11 years (Tdap) in different countries. Probably a few more years of data are needed to evaluate the real impact on infant disease before a universal recommendation could be considered.

Given this context, mathematical models of disease transmission contribute to a timely analysis of the impact of the new control strategies. In fact, an increasing number of papers on pertussis modelling have been reported [13-20]. In this work we present an age-structured compartmental model to describe the propagation of pertussis in Argentina and to assess the impact of vaccination schedule changes on infant disease control. The model explores scenarios for future interventions in countries with social, demographic and epidemiological similarities to Argentina. Thus a brief overview of the Argentinean epidemiological situation is given before a description of the mathematical model.

\section{Pertussis epidemiology in Argentina}

Argentina is a South American country with a population of 40091359 inhabitants, and a high life expectancy $(75.5$ years) and literacy rate $(97 \cdot 7 \%)$, comparable to developed countries. From the beginning of pertussis vaccination in the 1970s and until 1985, the Argentinean schedule against pertussis included three primary doses of diphtheria-tetanuswhole cell pertussis vaccine (DTw $\overline{\mathrm{P}}$ ) at ages $\overline{2}, 4$ and $\overline{6}$ months and a booster dose of DTwP at 18 months. In 1985, a fifth DTwP dose at age 5-6 years (school
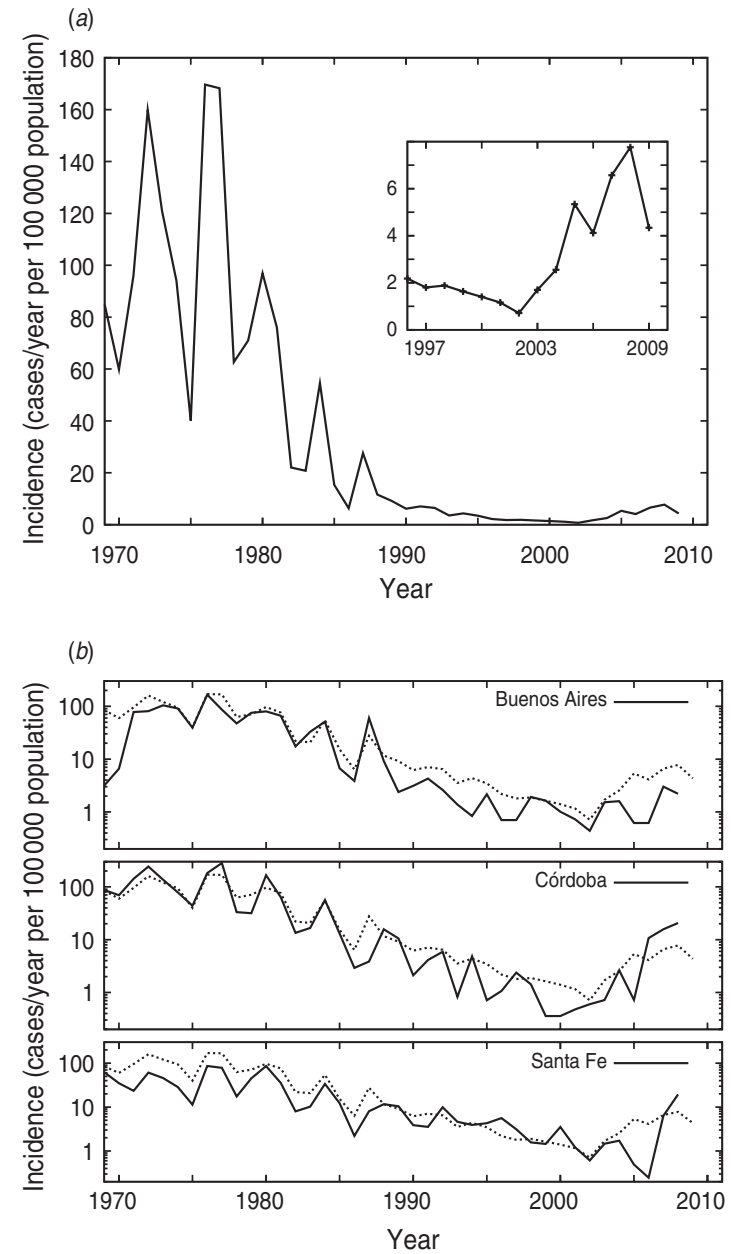

Fig. 1. (a) Pertussis incidence in Argentina during the 1969-2009 period. (b) Pertussis incidence in three Argentinean provinces. Pertussis incidence for the whole country is shown by dotted lines. (Source: SINAVE, Argentinean Ministry of Health.)

entry) was introduced. Since 2009, in accord with international recommendations [21], an acellular pertussis booster dose (TdaP) was included for 11-yearolds and healthcare workers. All pertussis vaccine doses are distributed free of charge to the population. The three-dose pertussis coverage is high, typically $90 \%$. In 2010 DTwP3 average coverage for Argentina was $92.8 \%$ [22]. However, this value is not uniform throughout the country because there are some regions with coverage lower than $80 \%$ [23]. The reported 18-month dose coverage is in general lower than the other doses. In 2010 the 11-year booster coverage was $57 \%$ [22]. In Argentina, as occurred in other countries, the introduction of mass immunization markedly decreased pertussis hospitalizations and deaths, reaching the lowest incidence levels in 2002 [23, 24] (Fig. 1a). Figure $1 a$ depicts the occurrence of 
pertussis outbreaks every 3-4 years, as was also reported for other countries [25-27]. These periodic outbreaks were also observed in different Argentinean districts in the vaccine era, and since the oscillations in incidence levels in the different districts are out of phase, the whole country oscillation becomes undetectable (Fig. 1 b). Since 2002 an increase in pertussis incidence has been observed, which was sustained until the present (2012). During 2011, 8323 suspected pertussis cases were notified in the National Surveillance System (SIVILA). From these total cases, 6821 were registered in infants aged $<1$ year. The majority of these cases corresponded to the more populated provinces of Argentina such as Buenos Aires, Córdoba and Santa Fe. From those cases, 1663 (19.98\%) were laboratory-confirmed pertussis cases. In 2010, 4981 cases were registered and 828 (16.62\%) were laboratory confirmed. Again most of the cases were confirmed in patients aged $<1$ year $(4217$ from 4981). The large proportion of cases recorded in infants was not unexpected, since pertussis is most severe in this age group. The number of pertussis deaths during the analysed period of 2011 was around 70 (this value was extracted from national and local reports), recorded mostly in infants aged $<1$ year. As expected, young unvaccinated infants were more likely to have a severe disease, to suffer complications, to require hospitalization, or to die than older children and adults.

\section{METHODS}

\section{Model}

We developed a deterministic age-structured compartmental model to evaluate the effects of health policies on pertussis incidence in infants, mainly in the $0-1$ year age group. The age discrimination allows us to simulate the application of vaccination doses at specific ages, as well as to explore the effect on pertussis transmission of different age profiles for the force of infection (FOI). The model described here is based on Hethcote's model designed in regard to pertussis in USA [15]. Here the population is stratified in nine epidemiological classes. Considering the severity of pertussis infection and consequently their infectivity, infected individuals were grouped into three classes: $I_{1}$ for severe infection, $I_{2}$ for mild infection, and $\mathrm{I}_{3}$ for weak infection. Individuals belonging to $I_{1}, I_{2}$, and $I_{3}$ have decreasing infectivity. Populations that are susceptible to acquiring the

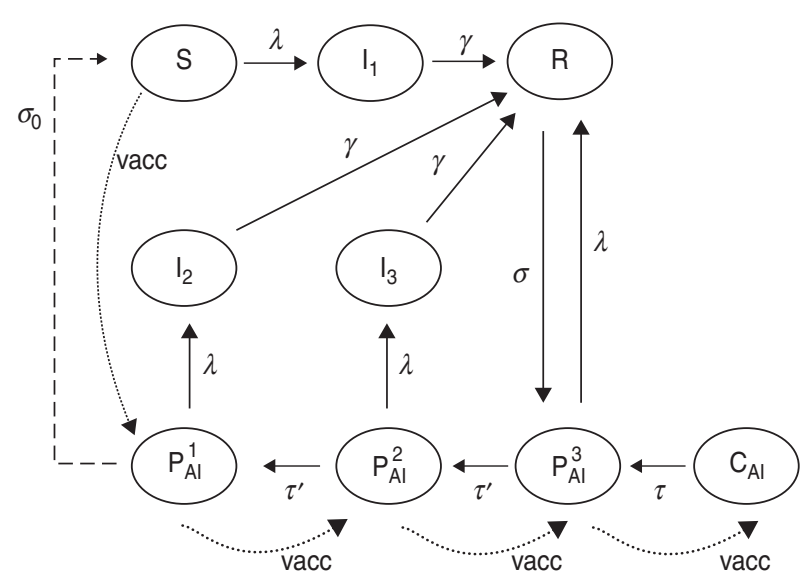

Fig. 2. Schematic representation of the epidemiological model.

infection are included in classes $\mathrm{S}, \mathrm{P}_{\mathrm{AI}}^{1}, \mathrm{P}_{\mathrm{AI}}^{2}, \mathrm{P}_{\mathrm{AI}}^{3}$, but while individuals in $\mathrm{S}$ class (fully susceptible) have no immunity to the infection, $\mathrm{P}_{\mathrm{AI}}$ classes include individuals with partial acquired immunity via vaccination or natural infection. Classes $\mathrm{R}$ (recovered) and $\mathrm{C}_{\mathrm{AI}}$ (complete acquired immunity) include individuals fully protected against the disease.

The dynamics of pertussis propagation is simulated by transferring individuals in different classes at corresponding specific transfer rates (Fig. 2). In this model, individuals are in the susceptible class when born, and remain there unless: (a) they become infectious through contact with an infected individual and enter the full symptomatic infective class $\left(\mathrm{I}_{1}\right)$, or (b) they acquire the lowest level of immunity through a vaccine dose and enter the $\mathrm{P}_{\mathrm{AI}}^{1}$ class. When receiving successive vaccination doses (indicated in Fig. 2 by dotted lines), individuals go through classes of increasing immunity and eventually reach the $\mathrm{C}_{\mathrm{AI}}$ class. Individuals in classes $\mathrm{P}_{\mathrm{AI}}^{1}$ and $\mathrm{P}_{\mathrm{AI}}^{2}$ may become infectious but they develop a less symptomatic illness entering class $\mathrm{I}_{2}$ (mild infection) or $\mathrm{I}_{3}$ (weak infection), respectively. We assume that infection fades in a time that is exponentially distributed around an average value $1 / \gamma$. After this time, individuals in infective classes $I_{1}, I_{2}$ or $I_{3}$ recover and enter class $R$. Individuals in $\mathrm{P}_{\mathrm{AI}}^{3}$ class acquire an infection but it is so weak that they do not become infective and so directly enter $\mathrm{R}$ class. The main effect of infection on $\mathrm{P}_{\mathrm{AI}}^{3}$ individuals is to boost their own immunity, contributing to herd immunity.

In this model we assume that immunity does not last lifelong. We consider that pertussis immunity is lost gradually. This effect was simulated by transferring individuals from the classes with complete 
Table 1. Parameter values for the different pertussis immunity durations considered in this study

\begin{tabular}{lrrrrrr}
\hline \hline Duration of pertussis immunity & $t_{\mathrm{N}}$ & $t_{\mathrm{V}}$ & $1 / \sigma$ & $1 / \tau$ & $1 / \tau^{\prime}$ & $1 / \sigma_{0}$ \\
\hline Short duration immunity (SDI) & 8 & 4 & 5 & 1 & $1 \cdot 5$ & 100 \\
Medium duration immunity (MDI) & 15 & 6 & 11 & 2 & 2 & 100 \\
Long duration immunity (LDI) & 20 & 10 & 14 & 4 & 3 & 100 \\
\hline \hline
\end{tabular}

$t_{\mathrm{N}}$ and $t_{\mathrm{V}}$ are the duration of immunity acquired naturally (through infection) or artificially (through vaccination), respectively. The rates $\sigma, \tau$ and $\tau^{\prime}$ are chosen to obtain $t_{\mathrm{N}}=1 / \sigma+2 / \tau^{\prime}, t_{\mathrm{V}}=1 / \tau+2 / \tau^{\prime}$. The rate at which immunized individuals become completely susceptible is controlled by the slow rate, $\sigma_{0}$. All the values in the table are in years.

immunity ( $\mathrm{R}$ and $\mathrm{C}_{\mathrm{AI}}$ ) to classes with decreasing degrees of immunity $\left(\mathrm{P}_{\mathrm{AI}}^{3} \rightarrow \mathrm{P}_{\mathrm{AI}}^{2} \rightarrow \mathrm{P}_{\mathrm{AI}}^{1}\right)$. Individuals in classes $\mathrm{R}$ or $\mathrm{C}_{\mathrm{AI}}$ have complete immunity against infection for a typical time $1 / \sigma$ or $1 / \tau$, respectively, in order to account for the difference of duration of complete immunity if it has been acquired via infection or vaccination. We assume that further loss of immunity occurs at the same rate $\tau^{\prime}$, independently of the way it has been acquired. This is a simplification introduced in our model with respect to the one used by Hethcote [15]. We argue that with a careful choice of parameters $\sigma, \tau$ and $\tau^{\prime}$ we may account for the main effects that introduce the difference between natural and artificially acquired loss of immunity. Like Hethcote we consider the possibility that after a very long time individuals eventually become fully susceptible. In the model this fact was included by transferring individuals from $\mathrm{P}_{\mathrm{AI}}^{1}$ to $\mathrm{S}$ at a rate $\sigma_{0}$. The nine epidemiological classes described above are divided into age groups. Thus, for fully susceptible individuals, for example, we define $\mathrm{S}_{i}(t)$ as the fraction of individuals in class $\mathrm{S}$, at time $t$, with age in the interval $\left(a_{i}, a_{i+1}\right)$. The FOI $\lambda_{i}$ is the rate at which susceptible or partially immune individuals of age group $i$ acquire infection. This is the only rate in our model that is not constant through time and depends on the fractions of infected individuals (which are dynamical variables of the model) through the expression:

$\lambda_{i}=\sum_{j} \beta_{i j} \mathrm{I}_{j}^{*} ; \quad \mathrm{I}_{j}^{*}=\mathrm{I}_{1 j}+\rho_{1} \mathrm{I}_{2 j}+\rho_{2} \mathrm{I}_{3 j}$

where $\beta_{i j}$ is the contact parameter matrix and $\mathrm{I}_{j}^{*}$ is the effective fraction of individuals of age $j$ in the population that is infective. Factors $\rho_{1}$ and $\rho_{2}$ are taken as smaller than 1 to consider that infected individuals in classes $I_{2}$ and $I_{3}$ are less infective than the ones in class $I_{1}$ as they have a milder cough. The dynamics of the model is described by a set of coupled ordinary differential equations discussed in the Appendix where a detailed description of the model is included. By solving these equations, the population of the different classes discriminated by age group as a function of time can be obtained.

\section{Model parameters}

We define a set of ages: $a_{i}=0,2,4,6,12,18$ months, yearly from 2 to 15 years; every 5 years from 15 to 55 years, and every 10 years from 55 to 75 years, which determines the $n_{\mathrm{A}}=30$ age groups: $\left(a_{i}, a_{i+1}\right)$. For infants aged $<18$ months the ages were chosen taking into account the local vaccination schedule. In this work the size of population is considered constant in time (see Appendix). Concerning mortality rates, $\mu_{i}$, in this work we assume that type I mortality (everybody dies at an age equal to life expectancy, see Appendix for details) is an acceptable approximation for Argentina (Supplementary Fig. S1, available online). Although it is well known that pertussis vaccination or infection does not provide lifelong immunity, the precise duration of protection is not known $[9,15,18]$. Because of that, three immunity parameterizations were considered here: SDI, MDI and LDI for short, medium or long duration of immunity, respectively, based on data from Wendelboe et al. [9]. It is assumed that the duration of natural immunity $\left(t_{\mathrm{N}}\right)$ is the time elapsed since an individual has recovered from infection (enters class $\mathrm{R}$ ) until reaching class $\mathrm{P}_{\mathrm{AI}}^{1}$ where the individual may acquire a mild infection. The duration of immunity acquired through vaccination $\left(t_{\mathrm{V}}\right)$ is the time elapsed since an individual has been completely immunized (enters class $\mathrm{C}_{\mathrm{AI}}$ ) until reaching class $\mathrm{P}_{\mathrm{AI}}^{1}$. Table 1 lists the values used for these parameters in the three immunity conditions considered. As in Hethcote's work, 
Table 2. Pertussis vaccination coverage for $C_{95}$ and $C_{80}$

\begin{tabular}{llllll}
\hline \hline \multicolumn{5}{l}{ Age of dose application } \\
\cline { 2 - 6 } & $2 \mathrm{mo}$. & $4 \mathrm{mo}$. & $6 \mathrm{mo}$. & $18 \mathrm{mo}$. & $6 \mathrm{yr}$ \\
\hline $\mathrm{C}_{95}$ & 0.95 & 0.95 & 0.95 & 0.85 & 0.95 \\
$\mathrm{C}_{80}$ & 0.80 & 0.80 & 0.80 & 0.70 & 0.80 \\
\hline \hline
\end{tabular}

The values of coverage included in the table are prior to the introduction of Tdap booster (at 11 years).

the efficacy of a pertussis vaccine dose (VE) was considered equal to 0.9 [15]. Taking into account that vaccine coverage in Argentina depends on the region [23], we considered both high vaccination coverage of $95 \%$ for the three-dose pertussis $\left(\mathrm{C}_{95}\right)$ and low vaccination coverage of $80 \%$ for the three-dose pertussis $\left(\mathrm{C}_{80}\right)$. In both cases, the 18-month dose coverage is lower than the other doses and was considered $85 \%$ and $70 \%$, respectively, reaching the three-dose coverage for the 6-year boost (Table 2). The transmission of infection takes place through contact between infected and susceptible (or partially immunized) individuals. Quantitatively, this process is controlled by expression (1) for the FOI $\lambda_{i}$. Individuals with different grades of infection contribute differently to $\lambda_{i}$. This contribution was taken into account with the parameters $\rho_{1}$ and $\rho_{2}$. In particular the values used were those proposed by Hethcote: $\rho_{1}=0.5, \rho_{2}=0.25$ [15]. The recovery rate is set as $\gamma=1 /(21$ days $)$ independently of the degree of infection. In order to determine $\beta_{i j}$ contact parameters, two different procedures were followed, which led us to consider three different sets of contact parameters.

\section{Procedure 1: Determination of contact parameters, $\beta_{i j}$, from the FOI and WAIFW (who acquires infection from whom) matrices}

In the standard procedure described by Anderson \& May [25], $\beta_{i j}$ is supposed to be symmetric among the age groups and some structure for matrix $\beta$ is assumed in such a way that it has only few $n_{\mathrm{B}}$ independent values. This structure is usually referred to as WAIFW matrix. If $n_{\mathrm{B}}$ values of $\lambda_{i}$ are known from epidemiological data, the given epidemiological model may be solved, the $\mathrm{I}_{j}^{*}$ at the stationary state of the system may be obtained, and equation (1) is inverted in order to obtain the $n_{\mathrm{B}}$ values that define $\beta_{i j}$. The details of this procedure and the minor modifications introduced by us in the present work are discussed in the Supplementary online material.
A common practice in this parameterization procedure has been to take $\lambda_{i}$ values from the pre-vaccine era and then use the $\beta_{i j}$ parameters obtained in the vaccine era assuming that the contact structure of the population has not changed significantly [15, 28]. There are, however, other models that parameterize $\beta_{i j}$ from the knowledge of FOI in the vaccine era $[16,29]$. A marked effort is currently made to obtain $\lambda_{i}$ values from empirical data when the immunological situation is much more complicated than in the prevaccine era [30]. In Argentina, there are no sufficiently accurate age-stratified data to estimate $\lambda_{i}$ values in the pre-vaccine or vaccine eras. Therefore, some standard $\lambda$ age profiles of the pre-vaccine (case A) and vaccine (case B) eras are considered and discussed below.

\section{FOI from the pre-vaccine era (case A)}

The FOI in the pre-vaccine era could in principle be taken from any country because the pertussis epidemiological situation, before massive vaccination was implemented, was supposed to be similar in different countries. On the contrary, vaccination introduces several new variables such as coverage of vaccine formulations, time since application and age cohorts that have been vaccinated, which should be carefully considered when extrapolating parameters from one place to another. The values used here (shown in Fig. 3a) were based on those reported by Hethcote [15] with a slight modification in order to fit them in the age groups of our model. The relevant feature of this FOI profile is the peak for the 5-10 years age group. The average age at first infection, $A_{1 \mathrm{~s}}$, estimated by our model (see Appendix) is between 4.4 and 5 years as expected for pertussis [25]. It is important to note that as the determination of $\beta_{i j}$ involves the solution of the epidemiological model, we obtain three sets of contact parameters (CP) one for each duration of immunity considered (SDI, MDI, LDI).

\section{FOI based on studies in the vaccine era (case B)}

In this case we follow a similar procedure as in case A but instead of taking the FOIs of the pre-vaccine era, we propose another procedure (see details in the Supplementary material). This procedure was developed in order that the FOI obtained with our model for the vaccine era shows some features recently observed in serological and demographic studies in several European countries [30]. These characteristics are: a peak of pertussis incidence for teenagers, 

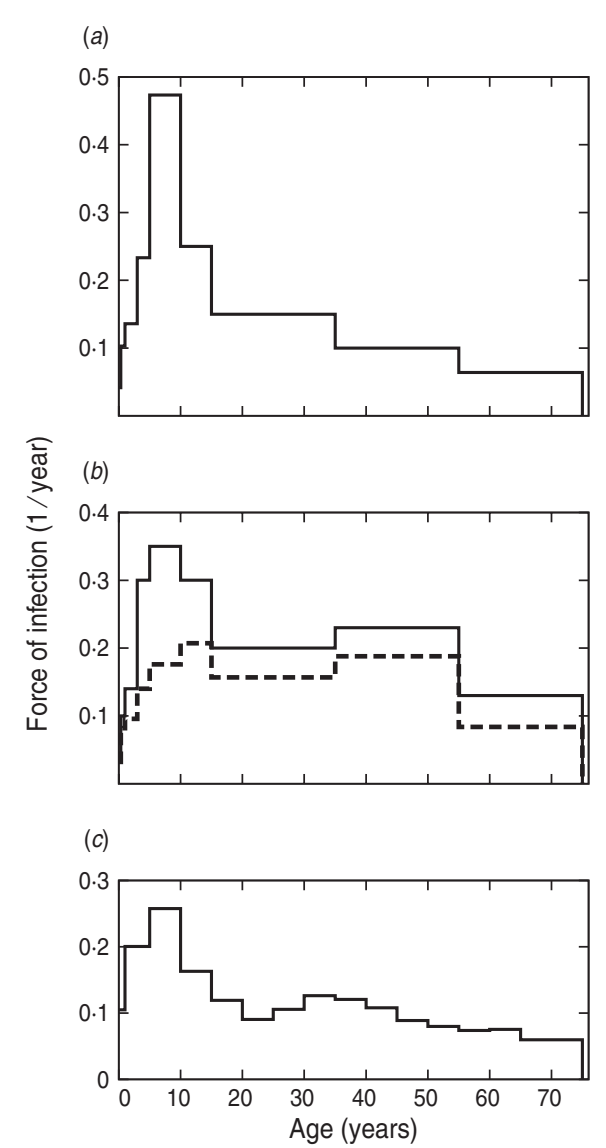

Fig. 3. Force of infection (FOI) for medium duration of immunity in the pre-vaccine era (-) and vaccine era (-- -) with $\mathrm{C}_{95}$ coverage. Data in panels $(a)$ and $(b)$ have been used to parameterize the model in procedure 1 , cases $\mathrm{A}$ and $\mathrm{B}$, respectively. Panel $(c)$ shows the FOI obtained with the model when procedure 2 is used to parameterize $\beta_{i j}$.

the presence of a second broad peak at around age 45 years, and the fact that both peaks mostly share the same height (with the exception of Italy). The FOI both in the pre-vaccine and vaccine eras are shown in Figure $3 b$. The higher values of the FOI assigned to adults in comparison to case $\mathrm{A}$, leads to higher values for the $\beta_{i j}$ parameters that describe contacts among adults aged $>35$ years.

\section{Procedure 2: Determination of $\boldsymbol{\beta}_{i j}$ from social contact patterns}

$\beta_{i, j}$ parameters could also be determined from direct estimation of social contacts among different age groups. If a measurement of the rate $c_{i j}$ of the social contacts per day of an individual in age group $i$ with individuals of age group $j$ were available, $\beta_{i j}$ could be approximated assuming that the probability of a contact being infective is the same for all age groups (see Supplementary material). An extensive study of social contacts in eight different European countries relevant for airborne or close-contact infectious diseases has been performed by Mossong and coworkers [31]. These authors found that the eight sets of $c_{i j}$ obtained in the analysed countries, present similar qualitative features. We have used the statistical weighted average of the $c_{i j}$ reported in Mossong et al. [31] to construct another possible contact pattern parameterization, even though the structure of social contacts in our country may present its intrinsic complexity. In Figure $3 c$ we show the FOI predicted from this procedure for the MDI case in the prevaccine era.

A detailed description of the assumptions and methodology used to determine $\beta_{i j}$ for procedures 1 and 2 , and the resulting values are presented in the Supplementary material.

\section{Epidemiological scenarios}

In summary, for the parameterization of the model we consider different possible values for the three sets of parameters that define duration of immunity, contact patterns among people of different age groups and vaccine coverage. In Table 3 the notation used for these parameters is included. From these parameters 18 scenarios in the vaccine era were defined: SDI$\mathrm{CP}_{1 \mathrm{~A}}-\mathrm{C}_{95}$, MDI-CP ${ }_{1 \mathrm{~A}}-\mathrm{C}_{95}$, LDI-CP ${ }_{1 \mathrm{~A}}-\mathrm{C}_{95}$, SDI-CP ${ }_{1 \mathrm{~B}^{-}}$ $\mathrm{C}_{80}, \ldots$ It is important to note that each scenario is not the combination of independent parameters. While in the case of SDI, MDI, LDI, $\mathrm{C}_{95}$ and $\mathrm{C}_{80}$ each defines a set of parameter values, each $\mathrm{CP}$ refers to the procedure used to determine $\beta_{i j}$, but in order to obtain $\beta_{i j}$ it is also necessary to give values to parameters that define the duration of immunity. For example, for $\mathrm{CP}_{1 \mathrm{~A}}$ we have three sets of $\beta_{i j}$, one for MDI, another for SDI and another for LDI.

\section{RESULTS}

Our study evaluates the impact of an adolescent booster dose (Tdap) on the most vulnerable $0-1$ year age group using an age compartmental deterministic model. For all the calculations here presented we considered that coverage for Tdap dose is $85 \%$ even though the current coverage is $57 \%$. This assumption was performed expecting that in the following years Tdap coverage will increase as was the case with the other vaccines introduced in the national immunization schedule. 
Table 3. Pertussis epidemiological scenario parameters

\begin{tabular}{lll}
\hline $\begin{array}{l}\text { Epidemiological features considered } \\
\text { to define scenarios }\end{array}$ & $\begin{array}{l}\text { Set of } \\
\text { parameters }\end{array}$ & $\begin{array}{l}\text { Values of } \\
\text { parameters: }\end{array}$ \\
\hline $\begin{array}{l}\text { Duration of immunity (DI) } \\
\text { SDI: Short }\end{array}$ & $\sigma, \tau, \tau^{\prime}, \sigma_{0}$ & Table 1 \\
MDI: Medium & & \\
LDI: Long & $\beta_{i j}$ & Tables S1, S2* \\
Contact parameters (CP) & & Tables S1, S2* \\
$\mathrm{CP}_{1 \mathrm{~A}}:$ from procedure 1 (case A) & Table S3* \\
$\mathrm{CP}_{1 \mathrm{~B}}:$ from procedure 1 (case B) & & Table 2 \\
$\mathrm{CP}_{2}:$ from procedure 2 & $p_{i}$ & \\
$\mathrm{Vaccine}^{*}$ coverage & & $\mathrm{C}_{95}: 95 \%$ for first three doses \\
$\mathrm{C}_{80}: 80 \%$ for first three doses & & \\
\hline \hline
\end{tabular}

* See Supplementary tables in the online Supplementary material.

Each combination of DI-CP vaccine coverage defines a specific 'scenario'.

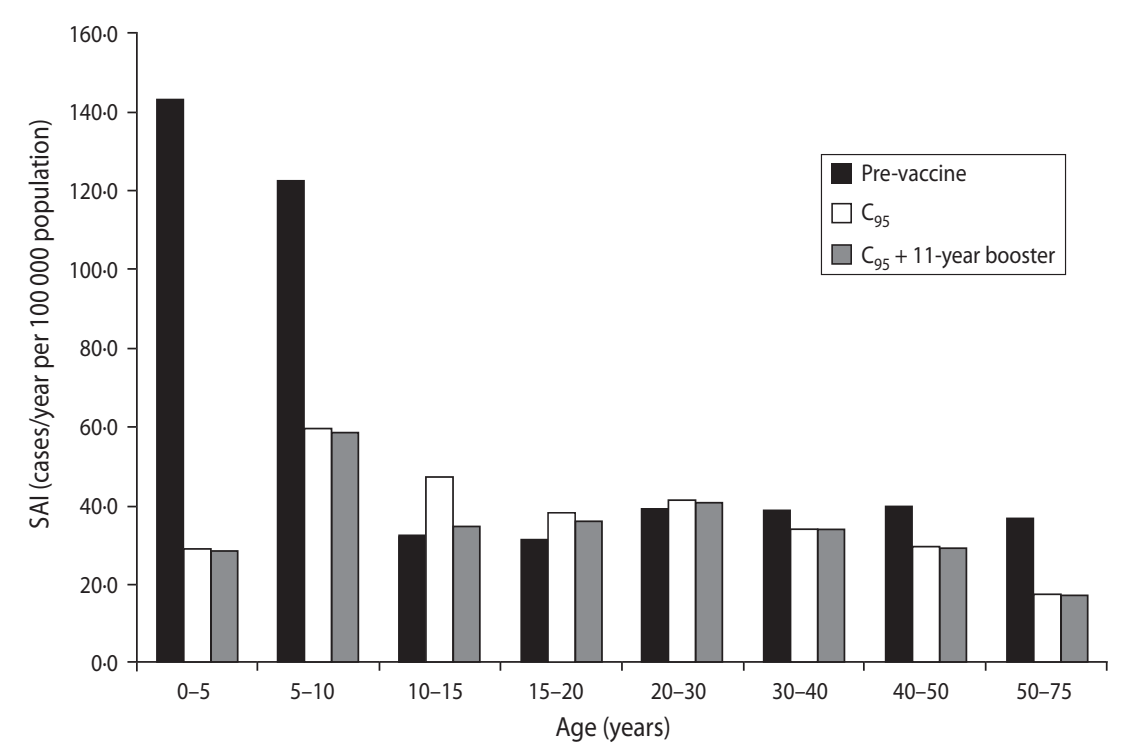

Fig. 4. Effect of $\mathrm{C}_{95}$ vaccination schedule on the age-specific pertussis incidence for the $\mathrm{CP}_{1 \mathrm{~A}}$-MDI scenario. Results considering an extra 11-year booster dose are also presented.

\section{Specific age incidences}

The major focus of our attention was on pertussis incidence of fully $\left(\mathrm{Inc}_{1}\right)$ and mild $\left(\mathrm{Inc}_{2}\right)$ symptomatic pertussis cases, which are the most risky classes (infected and infective). These incidences may be computed for each age group as follows:

$\operatorname{Inc}_{1 \mathrm{i}}=\lambda_{i} \mathrm{~S}_{i}, \quad \operatorname{Inc}_{2 \mathrm{i}}=\lambda_{i} \mathrm{P}_{\mathrm{AI} i}^{1}$

Inc $_{1}$, in an age interval $\Delta$, means the sum of $\operatorname{Inc}_{1 i}$ for all age groups contained in $\Delta$. Specific age incidence (SAI) in interval $\Delta$ is defined as $\left(\mathrm{Inc}_{1}+\mathrm{Inc}_{2}\right) / \Delta$. Inc and $\mathrm{Inc}_{2}$ depend on time through the dynamical variables $\lambda_{i} \mathrm{~S}_{i}, \mathrm{P}_{\mathrm{AI} i}^{1}$. However, all the results presented here and in the following section refer to the stationary values of incidences. We first focus on the $\mathrm{CP}_{1 \mathrm{~A}}$-MDI scenario since contacts among individuals are based on standard FOIs that have been widely used by different authors [15, 25]. Regarding loss of immunity, MDI parameters correspond to the mean reported values and are similar to the ones used by Hethcote $[15,32]$. In this scenario SAIs for different age groups were calculated both in the pre-vaccine era and in the vaccine era with or without the extra dose at age 11 years. The results obtained are shown in Figure 4. The figure shows that in the pre-vaccine era, 
Table 4. Effect of Tdap booster dosing on 0-1 year incidence

\begin{tabular}{|c|c|c|c|c|c|c|}
\hline \multirow[b]{2}{*}{ Scenario } & \multicolumn{2}{|c|}{$\begin{array}{l}\text { Without Tdap } \\
11 \text {-year booster }\end{array}$} & \multicolumn{2}{|c|}{$\begin{array}{l}\text { With Tdap } \\
11 \text {-year booster }\end{array}$} & \multicolumn{2}{|c|}{$\begin{array}{l}\text { Difference in incidence with and } \\
\text { without Tdap 11-year booster }(\%)\end{array}$} \\
\hline & $\mathrm{Inc}_{1}$ & $\mathrm{Inc}_{2}$ & $\operatorname{Inc}_{1}$ & $\mathrm{Inc}_{2}$ & $\mathrm{Inc}_{1}$ & $\mathrm{Inc}_{1}+\mathrm{Inc}_{2}$ \\
\hline $\mathrm{CP}_{1 \mathrm{~A}}-\mathrm{SDI}$ & $6 \cdot 6$ & $16 \cdot 7$ & $6 \cdot 4$ & $16 \cdot 3$ & $2 \cdot 8$ & $2 \cdot 6$ \\
\hline $\mathrm{CP}_{1 \mathrm{~A}}-\mathrm{MDI}$ & $7 \cdot 3$ & $15 \cdot 0$ & $7 \cdot 2$ & $14 \cdot 7$ & $2 \cdot 1$ & $2 \cdot 2$ \\
\hline $\mathrm{CP}_{1 \mathrm{~A}}-\mathrm{LDI}$ & $7 \cdot 3$ & $12 \cdot 4$ & $7 \cdot 0$ & $12 \cdot 0$ & $3 \cdot 3$ & $3 \cdot 7$ \\
\hline $\mathrm{CP}_{1 \mathrm{~B}}-\mathrm{SDI}$ & $7 \cdot 4$ & $21 \cdot 5$ & $7 \cdot 3$ & $21 \cdot 3$ & $2 \cdot 2$ & $1 \cdot 5$ \\
\hline $\mathrm{CP}_{1 \mathrm{~B}}-\mathrm{MDI}$ & $8 \cdot 8$ & $19 \cdot 3$ & $8 \cdot 7$ & $19 \cdot 1$ & $1 \cdot 0$ & $1 \cdot 1$ \\
\hline $\mathrm{CP}_{1 \mathrm{~B}}-\mathrm{LDI}$ & $9 \cdot 0$ & $16 \cdot 5$ & $8 \cdot 9$ & $16 \cdot 3$ & $1 \cdot 2$ & $1 \cdot 4$ \\
\hline $\mathrm{CP}_{2}-\mathrm{SDI}$ & $13 \cdot 6$ & $19 \cdot 6$ & $13 \cdot 2$ & $19 \cdot 2$ & $2 \cdot 4$ & $2 \cdot 4$ \\
\hline $\mathrm{CP}_{2}-\mathrm{MDI}$ & $10 \cdot 2$ & $13 \cdot 8$ & $9 \cdot 9$ & $13 \cdot 4$ & $3 \cdot 1$ & $3 \cdot 0$ \\
\hline $\mathrm{CP}_{2}$-LDI & $7 \cdot 8$ & $9 \cdot 9$ & $7 \cdot 5$ & $9 \cdot 4$ & $4 \cdot 0$ & $4 \cdot 1$ \\
\hline
\end{tabular}

$\mathrm{CP}$, Contact parameter; SDI, short duration immunity; MDI, medium duration immunity; LDI, long duration immunity. Incidences are given in cases/year per 100000 population.

pertussis was an infection which mainly affected children. After the introduction of pertussis vaccine the incidence of disease in children declined markedly, particularly in the $0-5$ years age group, where $\mathrm{Inc}_{1}+\mathrm{Inc}_{2}$ decreased by a factor of 5 . Although lower than in the pre-vaccine era, in the vaccine era a peak of pertussis incidence is detected in children in the $5-10$ years age group. Moreover, in this era the SAI of adolescents aged 10-15 years is higher than that of the pre-vaccine era, probably as a consequence of the shorter duration of the immunity conferred by vaccination than that acquired by natural infection (Table 1). These changes in the calculated SAI caused by vaccination are in agreement with those previously reported in other studies that employed similar parameters to analyse pertussis in USA [15] and in Rio de Janeiro (Brazil) [17]. Figure 4 shows that the addition of the Tdap 11-year booster decreases the incidence of this age group but it has no significant effect on infants. We have even found that regardless the scenario considered the effect of adding an 11-year booster is still very small for children (Supplementary Fig. S2, online). Moreover, even when the coverage of the first five doses was low $\left(\mathrm{C}_{80}\right)$ or the incidence in adolescents was particularly high, the inclusion of an extra Tdap booster would not improve the epidemiological situation of children (Supplementary Fig. S2).

\section{Incidences for infants aged $<1$ year}

We then evaluated the effect of adolescent booster on pertussis incidence in infants aged $<1$ year for all scenarios analysed as shown in Table 4 . We observed that beyond the differences in $\mathrm{Inc}_{1}$ and $\mathrm{Inc}_{2}$ in the scenarios considered, the effect of the addition of Tdap booster either on $\mathrm{Inc}_{1}, \mathrm{Inc}_{2}$ or $\mathrm{Inc}_{1}+\mathrm{Inc}_{2}$ sum, is $<5 \%$ in all cases. The effect of including the Tdap booster was also compared with the effect of improving the coverage of the first three doses. In Figure 5, the 0-1 year incidence $\left(\mathrm{Inc}_{1}+\mathrm{Inc}_{2}\right)$ values for the nine scenarios, for $\mathrm{C}_{95}$ and $\mathrm{C}_{80}$ coverage, are shown. It is clear that beyond the immunity duration or the contact scenario considered, improving vaccination coverage produces a significant decrease in $0-1$ year incidence (between $22 \%$ and $32 \%$ ) in comparison with the incorporation of the booster at age 11 years (below $5 \%$ ). These data indicate that increasing the coverage of the first dose (at 2, 4 and 6 months) would result in a substantial improvement in the control of this disease.

\section{Dynamical behaviour}

The deterministic compartmental model presented here predicts that the system will reach a stationary equilibrium state that represents the endemic phase of the disease. Any disturbance of this equilibrium generates pronounced oscillations in the dynamical variables of the model that are smoothly damped over time. In particular, the introduction of the Tdap booster produces an oscillatory behaviour of incidence that is shown in Figure 6. The figure shows the dynamical evolution of $\operatorname{Inc}_{1}(t)+\operatorname{Inc}_{2}(t)$ for 10 years for the 11-13 and $0-1$ years age groups. Two years after introduction of the booster, when incidences attained their minimum, the drop of incidence observed in the figure for the 11-13 years group is about $47 \%$, while for the $0-1$ year group it is about $13 \%$. 

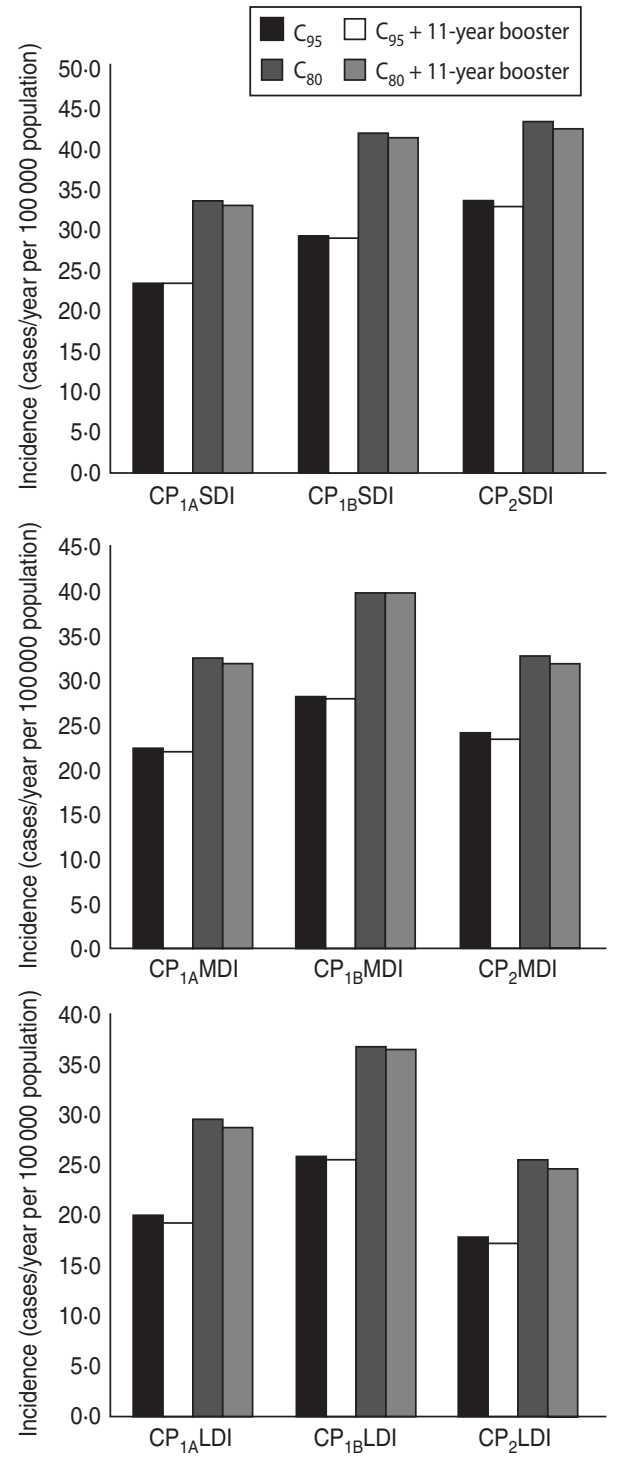

Fig. 5. Effect of adolescent booster dosing on $0-1$ year incidence $\left(\mathrm{Inc}_{1}+\mathrm{Inc}_{2}\right)$ in all scenarios for $\mathrm{C}_{95}$ and $\mathrm{C}_{80}$ coverage. Three durations of immunity were considered: short (upper panel), medium (middle panel) and long (lower panel).

Moreover, the incidence for the 11-13 years group oscillates around a new equilibrium value that is $43.5 \%$ lower than before the introduction of the booster. On the other side, in the $0-1$ year group incidence oscillates around a value very similar to the one it had before introduction of the booster (only $2 \%$ lower). The oscillatory behaviour of pertussis incidence has been predicted by both stochastic and deterministic epidemic models [14, 18, 33]. The periods of outbreaks predicted by our model in the three MDI scenarios are shown in Table 5 and lie within the expected values for pertussis [25-27]. In
Table 5. Inter-epidemic periods obtained for the MDI scenarios in the pre-vaccine and vaccine eras with $C_{95}$ or $C_{80}$ coverage

\begin{tabular}{llll}
\hline \hline & & \multicolumn{2}{l}{ Vaccine era } \\
\cline { 3 - 4 } Scenario & Pre-vaccine & $\mathrm{C}_{95}$ & $\mathrm{C}_{80}$ \\
\hline $\mathrm{CP}_{1 \mathrm{~A}}$-MDI & $2 \cdot 7$ & $4 \cdot 2$ & $4 \cdot 0$ \\
$\mathrm{CP}_{1 \mathrm{~B}}$-MDI & $3 \cdot 0$ & $3 \cdot 6$ & $3 \cdot 6$ \\
$\mathrm{CP}_{2}$-MDI & $3 \cdot 6$ & $5 \cdot 0$ & $5 \cdot 0$ \\
\hline \hline
\end{tabular}

CP, Contact parameter; MDI, medium duration immunity. Values given are in years.

particular, the observed period of 4 years for pertussis in Argentina (Fig. $1 b$ ) is well estimated by any of the scenarios considered. It should be noted that a precise prediction of the value of inter-epidemic period has remained very elusive as it depends on details in the model description, such as age dependence of the FOI [14], a stochastic treatment of the fluctuations in the network of contacts where the disease spreads [34] and the consideration of deterministic recovery $[34,35]$. Therefore, the results shown in Table 5 should not be interpreted as indicating that a certain scenario is better than another one because of a better prediction of the period of oscillations.

\section{Sensitivity of the previous results to different factors that may influence disease transmission}

Variation of $V E$

Adequate estimates of VE per dose are difficult, particularly in our country, because Argentina uses imported vaccines from different companies which varies from year to year. Because of that in this work we use the value of 0.9 estimated by Hethcote from USA epidemiological data [15]. However, as VE enters our model as a multiplying factor of vaccine coverage $p_{k}$, we may evaluate the effect of a lower VE when assessing the results for a lower coverage. Our results show that an adolescent booster has the same low effect in $0-1$ year incidence when the coverage is reduced from $95 \%$ to $80 \%$. Therefore, different VE values are not expected to modify the conclusions of our work.

\section{Predicted effect of maternal immunity transmission (MIT) in newborn protection}

Using our model we also analysed the effect of the transfer of immunity from mothers to infants in 


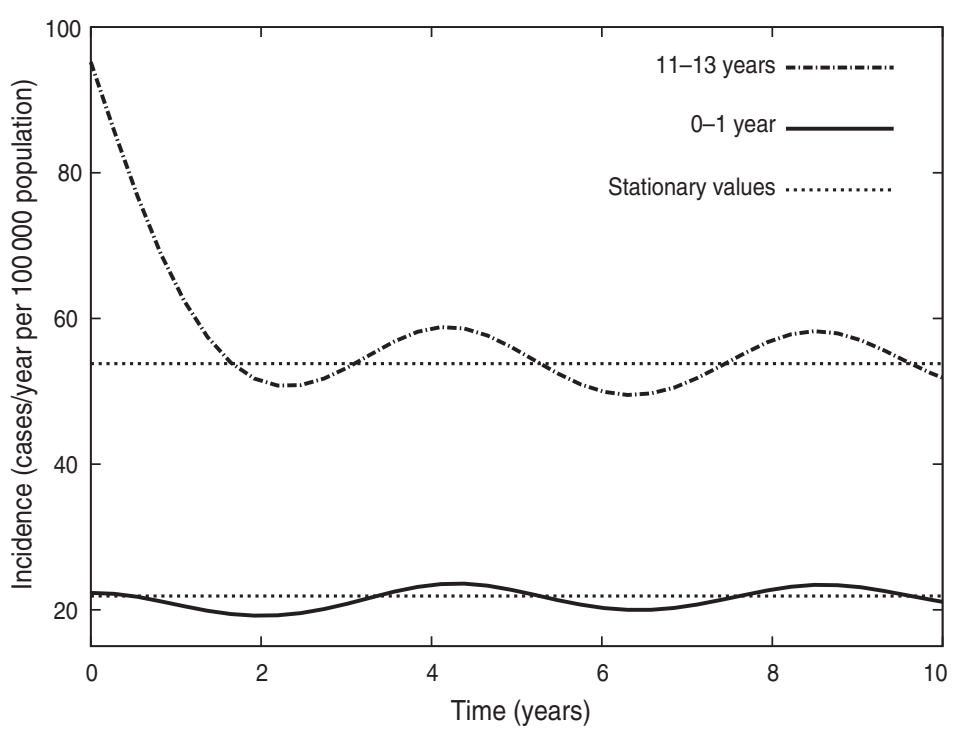

Fig. 6. Dynamical behaviour of incidence $\left(\mathrm{Inc}_{1}+\mathrm{Inc}_{2}\right)$ for the $\mathrm{CP}_{1 \mathrm{~A}}-\mathrm{MDI}$ scenario with $\mathrm{C}_{95}$ vaccination coverage after introduction of the 11-year booster. Time is measured from the booster introduction.

pertussis transmissibility. To this end, an extra class $\mathrm{X}_{0}$ was added to our model. This class includes individuals who acquired maternal antibodies and because of that, they would present mild symptomatology $\left(\mathrm{I}_{2}\right)$ if they become infected. The modifications introduced to the model equations when MIT is considered are described in the Appendix. Performing calculations for the $\mathrm{CP}_{1 \mathrm{~A}}$-MDI scenario, and assuming that maternal antibodies will last 2.5 years, we observed that MIT reduces the incidence Inc $_{1}$ in the $0-1$ year group by $10 \%$. If we consider adolescent booster dosing, the effect of boosting in reducing $0-1$ year incidence appears to be the same for this modified model that includes newborn protection as for the original model (typically $2 \%$ ). Based on these results and using the modified model that includes the $\mathrm{X}_{0}$ class, a rough approximation of the effect that mother booster dosing would have on infant incidence was estimated. If we consider that $50 \%$ of mothers are immunized, the calculated reduction for Inc $_{1}$ would lie around $33 \%$. It should be noted that although this reduction in $\mathrm{Inc}_{1}$ is associated directly with an equal increase in $\mathrm{Inc}_{2}$, it is a desirable effect since the severe form of the disease in children is reduced. In fact, the decrease of the full infection in the most vulnerable population with highest risk of hospitalization and death, is one of the goals pursued by the health system. The results presented in this section agree with the recommendations recently made by the ACIP of the USA CDC related to the use of Tdap vaccine in pregnant women [12].

\section{Analysis of the effect of immune memory on pertussis} transmission

There is evidence that immune memory cells exposed again to an antigen through infection or a complete vaccination scheme exhibit a more efficient response than naive cells. Therefore it is reasonable to suppose that a pertussis booster vaccination in individuals who have had a previous pertussis infection or at least three vaccine doses would confer the highest degree of immunity. We considered this effect in our model sending those individuals that exhibit a more efficient response directly to class $\mathrm{C}_{\mathrm{AI}}$ when a booster is applied independently of their presence in class $\mathrm{P}_{\mathrm{AI}}^{1}$, $\mathrm{P}_{\mathrm{AI}}^{2}$ or $\mathrm{P}_{\mathrm{AI}}^{3}$. These modifications were introduced in our model (see Appendix for details) and the computations of incidence for $\mathrm{CP}_{1 \mathrm{~A}}$-MDI scenarios repeated. The results (Table 6) point out that, even though the 11-year dose is predicted to be more effective by this modified model, it still shows a low impact on young infant incidence (below $5 \%$ ). In any case, the introduction of the adolescent booster in the vaccination schedule displays a minor effect in reducing incidence in the $0-1$ year group. This memory effect was also considered by other authors in SIR-based models including three types of partial immunity classes: removed, vaccinated and waning immunity classes [32].

Another way in which immune memory may affect pertussis transmission has recently been analysed by Lavine et al. [19]. These authors considered in their 
Table 6. Comparative analysis of the effect of Tdap booster dosing on the 0-1 year incidence simulated using unmodified and modified models

\begin{tabular}{|c|c|c|c|c|c|c|c|}
\hline & & \multicolumn{2}{|c|}{ Without boost } & \multicolumn{2}{|c|}{ With boost (at 11 years) } & \multicolumn{2}{|c|}{ Percent change } \\
\hline & & $\mathrm{Inc}_{1}$ & $\mathrm{Inc}_{2}$ & $\mathrm{Inc}_{1}$ & $\mathrm{Inc}_{2}$ & $\mathrm{Inc}_{1}$ & $\mathrm{Inc}_{1}+\mathrm{Inc}_{2}$ \\
\hline $\mathrm{C}_{95}$ & $\begin{array}{l}\text { Unmodified model } \\
\text { Modified model }\end{array}$ & $\begin{array}{l}7 \cdot 3 \\
6 \cdot 9\end{array}$ & $\begin{array}{l}15 \\
14 \cdot 1\end{array}$ & $\begin{array}{l}7 \cdot 2 \\
6 \cdot 6\end{array}$ & $\begin{array}{l}14 \cdot 7 \\
13 \cdot 4\end{array}$ & $\begin{array}{l}2 \cdot 1 \\
5\end{array}$ & $\begin{array}{l}2 \cdot 2 \\
5 \cdot 4\end{array}$ \\
\hline $\mathrm{C}_{80}$ & $\begin{array}{l}\text { Unmodified model } \\
\text { Modified model }\end{array}$ & $\begin{array}{r}10 \cdot 2 \\
9 \cdot 9\end{array}$ & $\begin{array}{l}22 \cdot 1 \\
21 \cdot 4\end{array}$ & $\begin{array}{l}10 \\
9 \cdot 6\end{array}$ & $\begin{array}{l}21 \cdot 7 \\
20 \cdot 7\end{array}$ & $\begin{array}{l}1 \cdot 5 \\
3\end{array}$ & $\begin{array}{l}1 \cdot 6 \\
3 \cdot 2\end{array}$ \\
\hline
\end{tabular}

Calculations were performed for the $\mathrm{CP}_{1 \mathrm{~A}}$-MDI scenario with $\mathrm{C}_{95}$ or $\mathrm{C}_{80}$ coverage.

In the modified model immunity memory effects are taken into account by subdividing classes $\mathrm{P}_{\mathrm{AI}}^{1}$ and $\mathrm{P}_{\mathrm{AI}}^{2}$ (see Appendix for more details).

transmission model that individuals could receive frequent natural boosters when exposed to a small number of bacteria. This possibility was also considered in our model taking a rate $\kappa \lambda$ instead of $\lambda$ for individuals that go from $\mathrm{P}_{\mathrm{AI}}^{3}$ to $\mathrm{R}$ class (see Fig. 2). When considering the $\mathrm{CP}_{2}$-MDI scenario taking $\kappa=2$, our results show that in the vaccine era $\left(\mathrm{C}_{95}\right)$ the system presents pronounced epidemic outbreaks similar to those obtained by Lavine et al. for high coverage [19] with no stationary state. Even when the dynamical description of the system predicted by our model when considering this boosting effect is very different, the values of mean incidence for infants are very similar. In particular, the effect of adding the 11-year booster in infants implies a $3 \%$ reduction of $0-1$ year incidence, as we have previously shown for our model (see Table 4). A detailed study of the consequences of introducing this effect in our model of pertussis transmission is beyond the scope of the present work and will be addressed elsewhere.

\section{DISCUSSION}

With the recommended application of the Tdap booster in adolescents, not only a reduction in adolescent pertussis incidence is expected but also an indirect benefit for infants could be achieved. This last objective seems to be feasible since the immunization of adolescents would remove at least in part the source of infection for infants. However, since currently there is no evidence to support this rationale, it is difficult to produce a universal recommendation to extend vaccination programmes to adolescents. Given this situation and to promptly assess the impact of the adolescent booster in infants, we designed a deterministic age-structured compartmental model based on two assumptions regarding pertussis immunity and transmission that are largely agreed upon: immunity has an important effect on the severity of disease, and immunity wanes over time. In this sense, the population in our model is stratified in nine classes (Fig. 2). With this model different possible epidemiological scenarios $(n=18)$ were explored since there are uncertainties in some parameters such as the rate of infectious contacts between people of different ages and also the exact duration of protective immunity.

In the 18 scenarios considered we could reproduce not only the drop in the incidence of children caused by the massive use of pertussis vaccine but also the fact that the SAI of the 10-15 years group was higher in the vaccine era than in the pre-vaccine era. These features reproduced here were evidenced by epidemiological studies in several countries [36] and different models $[15,17]$. It is important to note, however, that the magnitudes of the vaccine effects calculated here do not correlate with the data reported, probably due to underreporting of the disease. The general consensus is that the reported cases are probably much lower than the true incidence of pertussis. Because of this underreporting, the total pertussis incidence in the vaccine era predicted by the model, are much higher than those reported by national surveillance systems. The magnitude of the observed incidence reduction in Argentina due to vaccination (Fig. 1) is predicted by the model only for the incidence of fully symptomatic cases, Inc $\mathrm{I}_{1}$. This is the case, for example, for the calculated value of $\mathrm{Inc}_{1}$ in the $0-5$ years age group which is reduced by a factor of 75 from the pre-vaccine to the vaccine endemic equilibrium in the $\mathrm{CP}_{1 \mathrm{~A}}-\mathrm{MDI}-\mathrm{C}_{95}$ scenario. This agreement between the reported data and the calculated values of children $\left(\mathrm{Inc}_{1}\right)$ was also found in simulations of pertussis in USA [15] and supports 
the idea that the health system mainly detects child fully-symptomatic cases. The above-mentioned underreporting would be more pronounced in adolescents or adults where normal prolonged cough may be the only clinical feature. This underreporting makes it difficult to use notified data to decide which of the 18 scenarios is the best fit to reality. Following this rationale, different scenarios were considered to perform our analysis.

To evaluate the effect of the incorporation of the 11-year Tdap in the National Vaccination Schedule, we performed calculations for all considered scenarios. The results obtained showed that the 11-year Tdap booster decreases the incidence of the disease in this age group. This result is in agreement with the reported [37, 38] and predicted [29] data. When the effect of the introduction of an adolescent booster in infants was analysed, our results showed that the Tdap booster also reduces incidence in infants but to a very low extent. The calculated decrease does not exceed $5 \%$ for any of the scenarios considered. Since none of the variations included in the epidemiological scenarios leads to a reduction higher than $5 \%$, the prediction of the model proves to be robust. This result is in agreement with those reported by other authors using different models and parameterizations to obtain similar results $[19,29]$. Moreover, in some stochastic models in which it is considered that pertussis immunity is not lost, the reinforcement at 11 years does not impact in reducing the incidence in infants [20]. All these studies suggest that widespread adolescent vaccinations may not bring about the reduction in pertussis cases some had hoped. This could be explained at least in part by the relatively low weight that adolescents have as a source of infection in infants [39-41]. In fact, a recent work shows that adolescents exhibit different seasonal pertussis peaks compared to infants, suggesting that children and adults (possibly parents), not adolescents, are the main sources of infant infection [42]. The outcome of immunization of the relatively low fraction of adolescents that is a source of infant infection could be even lower because of Tdap efficacy, coverage and duration of immunity which do not ensure that the whole adolescent population is protected by the booster. This rationale supports the results predicted by our model.

Importantly, using our model we were able to demonstrate that, in countries such as Argentina where some regions have relatively low vaccine coverage, improving coverage of the first dose has a more beneficial impact in reducing incidence in infants than the vaccination of adolescents. In fact, a key outcome of our study is that increasing coverage of the first dose (at 2, 4 and 6 months) from $80 \%$ to $95 \%$, would result in a substantial reduction on infant incidence in all scenarios considered (between $22 \%$ and $32 \%$ ). However, in countries with a high coverage, a reduction of $5 \%$ in incidence could be assessed as positive. In this sense, Rosenbaum, using an agestructured compartmental deterministic model found that among several proposed vaccination strategies for The Netherlands (where coverage of first dose is $96 \%$ ) the optimum one is the addition of 12-year booster dose which produces a $5.5 \%$ reduction in infant infection [29]. Beyond the high vaccination coverage that could be achieved in a country, there are other strategies that may be better than vaccination in adolescents. In this sense modifications performed in our model evidence that vaccination of mothers during pregnancy would be a more effective strategy to protect infants than vaccination of adolescents. These results are consistent with recent widespread recommendations on vaccination of mothers after 20 weeks of gestation [12].

In summary, in this work we evaluate the Tdap booster impact on infant incidence considering some features relevant to pertussis transmission. We worked in the framework of deterministic compartmental age-structured models and have studied several epidemiological scenarios covering a wide range and combination of parameters. The impact of the Tdap booster on infants was also assessed by modifying our model in order to include the effect of transmission of mother immunity and the memory immune response of individuals. The results obtained showed that our predictions concerning the effect of Tdap booster in infants are robust. It is important to mention, however, that there are some other aspects of pertussis transmission that we have not explored as they introduce further complexities or they exceed the framework of our model and will be considered in depth in future work. This is the case of stochastic effects, heterogeneity of contacts because of social membership or regionality, changes in circulating bacteria, and several correlations that could be introduced properly in an agent-based model.

\section{SUPPLEMENTARY MATERIAL}

For supplementary material accompanying this paper visit http://dx.doi.org/10.1017/S0950268812001380. 


\section{ACKNOWLEDGEMENTS}

A. Melgarejo, A. Lodeiro, A. Graieb and I. Miceli are acknowledged for fruitful discussions. We thank Celina Castuma and M. Quiroga for the English revision. This work was supported by Agencia Nacional de Promoción Científica y Tecnológica-ANCPyT, Comisión de Investigaciones Científicas de Buenos Aires-CICBA and Consejo Nacional de Investigaciones Científicas y Tecnológicas-CONICET (Argentina) grants to D.F.H. and G.F. G.F. and P.B. are members of the Scientific Career of CONICET. D.F.H. is a member of the Scientific Career of CICBA.

\section{DECLARATION OF INTEREST}

None.

\section{REFERENCES}

1. Anon. Pertussis - United States, 1997-2000. Morbidity and Mortality Weekly Report 2002; 51: 73-76.

2. WHO. Pertussis vaccines: WHO position paper. Weekly Epidemiological Record 2010; 85: 385-400.

3. Baron S, et al. Epidemiology of pertussis in French hospitals in 1993 and 1994: thirty years after a routine use of vaccination. Pediatric Infectious Disease Journal 1998; 17: 412-418.

4. de Melker HE, et al. Pertussis in The Netherlands: an outbreak despite high levels of immunization with whole-cell vaccine. Emerging Infectious Diseases 1997; 3: 175-178.

5. Hozbor D, et al. Pertussis epidemiology in Argentina: trends over 2004-2007. Journal of Infection 2009; 59: 225-231.

6. de Melker HE, et al. The incidence of Bordetella pertussis infections estimated in the population from a combination of serological surveys. Journal of Infection 2006; 53: 106-113.

7. Mooi FR. Bordetella pertussis and vaccination: the persistence of a genetically monomorphic pathogen. Infection, Genetics and Evolution 2010; 10: 36-49.

8. Kallonen T, He Q. Bordetella pertussis strain variation and evolution postvaccination. Expert Review of Vaccines $2009 ; \mathbf{8}$ : 863-875.

9. Wendelboe AM, et al. Duration of immunity against pertussis after natural infection or vaccination. Pediatric Infectious Disease Journal 2005; 24: S58-61.

10. Yih WK, et al. The increasing incidence of pertussis in Massachusetts adolescents and adults, 1989-1998. Journal of Infectious Disease 2000; 182: 1409-1416.

11. Forsyth KD, et al. Prevention of pertussis: recommendations derived from the second Global Pertussis Initiative roundtable meeting. Vaccine 2007; 25: 2634-2642.

12. CDC. Updated recommendations for use of tetanus toxoid, reduced diphtheria toxoid and acellular pertussis vaccine (Tdap) in pregnant women and persons who have or anticipate having close contact with an infant aged $<12$ months-Advisory Committee on Immunization Practices (ACIP), 2011. Morbidity and Mortality Weekly Reports 2011; 60: 1424-1426.

13. Anderson RM, May RM. Age-related changes in the rate of disease transmission: implications for the design of vaccination programmes. Journal of Hygiene 1985; 94: $365-436$.

14. Grenfell BT, Anderson RM. Pertussis in England and Wales: an investigation of transmission dynamics and control by mass vaccination. Proceedings of the Royal Society of London, Series B 1989; 236: 213-252.

15. Hethcote HW. An age-structured model for pertussis transmission. Mathematical Biosciences 1997; 145: 89136.

16. van Boven M, et al. Waning immunity and sub-clinical infection in an epidemic model: implications for pertussis in The Netherlands. Mathematical Biosciences 2000; 164: 161-182.

17. Luz PM, et al. A modelling analysis of pertussis transmission and vaccination in Rio de Janeiro, Brazil. Epidemiology and Infection 2006; 134: 850-862.

18. Wearing HJ, Rohani P. Estimating the duration of pertussis immunity using epidemiological signatures. PLoS Pathogens 2009; 5: e1000647.

19. Lavine JS, King AA, Bjornstad ON. Natural immune boosting in pertussis dynamics and the potential for long-term vaccine failure. Proceedings of the National Academy of Sciences USA 2011; 108: 7259-7264.

20. Rohani P, Zhong X, King AA. Contact network structure explains the changing epidemiology of pertussis. Science 2010; 330: 982-985.

21. Forsyth KD, et al. Pertussis immunization in the Global Pertussis Initiative International Region Recommended Strategies and Implementation Considerations. Pediatric Infectious Disease Journal 2005 ; 24: S93-S97.

22. Argentinean Health Ministry database. (http://msal. gov.ar/htm/site/pdf/epidemiologia/boletin-semanal/ BoletinIntegradoDeVigilanciaVersionVF_SE34.pdf). Accessed 10 May 2012.

23. Argentinean Health Ministry database. (http://msal.gov. $\mathrm{ar} / \mathrm{htm} / \mathrm{site} / \mathrm{pdf} /$ AnexoVII_PAI-03-09.pdf). Accessed 10 May 2012.

24. Riva Posse CA, Miceli IN. Pertussis: its evolution in Argentina at the end of the twentieth century. Medicina (Buenos Aires) 2005; 65: 7-16.

25. Anderson R, May R. Infectious Diseases of Humans: Dynamics and Control. Oxford: Oxford University Press, 1991.

26. Broutin $\mathbf{H}$, et al. Large-scale comparative analysis of pertussis population dynamics: periodicity, synchrony, and impact of vaccination. American Journal of Epidemiology 2005; 161: 1159-1167.

27. Broutin $\mathbf{H}$, et al. Impact of vaccination and birth rate on the epidemiology of pertussis: a comparative study in 64 countries. Proceedings of the Royal Society of London, Series B, 2010; 277: 3239-3245.

28. Van Rie A, Hethcote HW. Adolescent and adult pertussis vaccination: computer simulations of five new strategies. Vaccine 2004; 22: 3154-3165. 
29. Rozenbaum MH, et al. Modelling the impact of extended vaccination strategies on the epidemiology of pertussis. Epidemiology and Infection. Published online: 24 November 2011. doi:10.1017/S0950268811002354.

30. Kretzschmar M, Teunis PF, Pebody RG. Incidence and reproduction numbers of pertussis: estimates from serological and social contact data in five European countries. PLoS Medicine 2010; 7: e1000291.

31. Mossong $\mathbf{J}$, et al. Social contacts and mixing patterns relevant to the spread of infectious diseases. PLoS Medicine 2008; 5: e74.

32. Hethcote HW. Simulations of pertussis epidemiology in the United States: effects of adult booster vaccinations. Mathematical Biosciences 1999; 158: 47-73.

33. Rohani P, Earn D, Grenfell BT. Opposite patterns of synchrony in sympatric disease metapopulations. Science 1999; 286: 968.

34. Simoes M, Telo da Gama MM, Nunes A. Stochastic fluctuations in epidemics on networks. Journal of the Royal Society Interface 2008; 5: 555-566.

35. Gonçalves S, Abramson G, Gomes MFC. Oscillations in SIRS model with distributed delays. European Physical Journal B 2011 ; 81: 363-371.
36. Brooks DA, Clover R. Pertussis infection in the United States: role for vaccination of adolescents and adults. Journal of the American Board of Family Medicine 2006; 19: 603-611.

37. Quinn HE, McIntyre PB. The impact of adolescent pertussis immunization, 2004-2009: lessons from Australia. Bulletin of the World Health Organization $2011 ; 89: 666-674$.

38. Skoff TH, et al. Early Impact of the US Tdap vaccination program on pertussis trends. Archives of Pediatrics and Adolescent Medicine 2012; 166: 344-349.

39. Jardine A, et al. Who gives pertussis to infants? Source of infection for laboratory confirmed cases less than 12 months of age during an epidemic, Sydney, 2009. Communicable Diseases Intelligence 2010; 34: 116-121.

40. Bisgard KM, et al. Infant pertussis: who was the source? Pediatric Infectious Disease Journal 2004; 23: 985-989.

41. Wendelboe AM, et al. Transmission of Bordetella pertussis to young infants. Pediatric Infectious Disease Journal 2007; 26: 293-299.

42. Lavine JS, et al. Short-lived immunity against pertussis, age-specific routes of transmission, and the utility of a teenage booster vaccine. Vaccine 2012; 30: 544-551.

\section{APPENDIX}

\section{Mathematical description of the model}

In our model individuals are assigned to one of the nine epidemiological classes $\mathrm{S}, \mathrm{P}_{\mathrm{AI}}^{1}, \mathrm{P}_{\mathrm{AI}}^{2}, \mathrm{P}_{\mathrm{AI}}^{3}, \mathrm{C}_{\mathrm{AI}}, \mathrm{I}_{1}, \mathrm{I}_{2}, \mathrm{I}_{3}$, and $\mathrm{R}$, based on their immunity to pertussis and infection status. We also define an age set: $a_{0}, a_{1}, a_{2}, \ldots$, and divide the population into age groups, where the age of the individuals in age group ' $i$ ' is in the interval: $\left(a_{i}, a_{i+1}\right)$. The epidemiological state of the population is specified by the fraction of individuals (with respect to the total population) in each one of the epidemiological classes: $\mathrm{S}_{i}, \mathrm{P}_{\mathrm{AI} i}^{1}, \mathrm{P}_{\mathrm{AI} i}^{2}, \mathrm{P}_{\mathrm{AI} i}^{3}, \mathrm{C}_{\mathrm{AI} i}, \mathrm{I}_{1 i}, \mathrm{I}_{2 i}, \mathrm{I}_{3 i}, \mathrm{R}_{i}$, for each one of the $n_{\mathrm{A}}$ age groups, $i=0, \ldots, n_{\mathrm{A}}-1$. The dynamical evolution of these variables monitors the propagation of the disease and is described by the following set of ordinary first-order coupled differential equations:

$$
\begin{aligned}
& \frac{\mathrm{d} \mathrm{S}_{i}}{\mathrm{~d} t}=-\lambda_{i} \mathrm{~S}_{i}+\sigma_{0} \mathrm{P}_{\mathrm{AI} i}^{1}-\mu_{i} \mathrm{~S}_{i}+c_{i-1}\left(1-\operatorname{vacc}_{i}\right) \mathrm{S}_{i-1}-c_{i} \mathrm{~S}_{i}+\delta_{i 0} B \\
& \frac{\mathrm{d}_{\mathrm{AI} i}^{1}}{\mathrm{~d} t}=-\lambda_{i} \mathrm{P}_{\mathrm{AI} i}^{1}-\sigma_{0} \mathrm{P}_{\mathrm{A} I}^{1}+\tau^{\prime} \mathrm{P}_{\mathrm{AI} i}^{2}-\mu_{i} \mathrm{P}_{\mathrm{AI} i}^{1}+c_{i-1}\left(1-\operatorname{vacc}_{i}\right) \mathrm{P}_{\mathrm{AI} i-1}^{1}-c_{i} \mathrm{P}_{\mathrm{A} I i}^{1}+c_{i-1} \operatorname{vacc}_{i} \mathrm{~S}_{i-1} \\
& \frac{\mathrm{d} \mathrm{P}_{\mathrm{AI} i}^{2}}{\mathrm{~d} t}=-\lambda_{i} \mathrm{P}_{\mathrm{AI} i}^{2}-\tau^{\prime} \mathrm{P}_{\mathrm{AI} i}^{2}+\tau^{\prime} \mathrm{P}_{\mathrm{AI} i}^{3}-\mu_{i} \mathrm{P}_{\mathrm{AI} i}^{2}+c_{i-1}\left(1-\operatorname{vacc}_{i}\right) \mathrm{P}_{\mathrm{AI} i-1}^{2}-c_{i} \mathrm{P}_{\mathrm{AI} i}^{2}+c_{i-1} \operatorname{vacc}_{i} \mathrm{P}_{\mathrm{AI} i-1}^{1} \\
& \frac{\mathrm{d} \mathrm{P}_{\mathrm{AI} i}^{3}}{\mathrm{~d} t}=-\lambda_{i} \mathrm{P}_{\mathrm{AI} i}^{3}-\tau^{\prime} \mathrm{P}_{\mathrm{AI} i}^{3}+\tau \mathrm{C}_{\mathrm{AI} i}-\mu_{i} \mathrm{P}_{\mathrm{AI} i}^{3}+\sigma \mathrm{R}+c_{i-1}\left(1-\operatorname{vacc}_{i}\right) \mathrm{P}_{\mathrm{AI} i-1}^{3}-c_{i} \mathrm{P}_{\mathrm{AI} i}^{3}+c_{i-1} \operatorname{vacc}_{i} \mathrm{P}_{\mathrm{AI} i-1}^{2} \\
& \frac{\mathrm{d} \mathrm{C}_{\mathrm{AI} i}}{\mathrm{~d} t}=-\tau \mathrm{C}_{\mathrm{AI} i}-\mu_{i} \mathrm{C}_{\mathrm{AI} i}+c_{i-1} \mathrm{C}_{\mathrm{AI} i-1}-c_{i} \mathrm{C}_{\mathrm{AI} i}+c_{i-1} \operatorname{vacc}_{i} \mathrm{P}_{\mathrm{AI} i-1}^{3} \\
& \frac{\mathrm{dI}}{\mathrm{d} t}=\lambda_{i} \mathrm{~S}_{i}-\gamma \mathrm{I}_{1 i}-\mu_{i} \mathrm{I}_{1 i}+c_{i-1} \mathrm{I}_{1 i-1}-c_{i} \mathrm{I}_{1 i} \\
& \frac{\mathrm{d} \mathrm{I}_{2 i}}{\mathrm{~d} t}=\lambda_{i} \mathrm{P}_{\mathrm{A} \mathrm{I} i}^{1}-\gamma \mathrm{I}_{2 i}-\mu_{i} \mathrm{I}_{2 i}+c_{i-1} \mathrm{I}_{2 i-1}-c_{i} \mathrm{I}_{2 i} \\
& \frac{\mathrm{dI} \mathrm{I}_{3 i}}{\mathrm{~d} t}=\lambda_{i} \mathrm{P}_{\mathrm{A} \mathrm{I} i}^{2}-\gamma \mathrm{I}_{3 i}-\mu_{i} \mathrm{I}_{3 i}+c_{i-1} \mathrm{I}_{3 i-1}-c_{i} \mathrm{I}_{3 i} \\
& \frac{\mathrm{d} \mathrm{R}_{i}}{\mathrm{~d} t}=\lambda_{i} \mathrm{P}_{\mathrm{AI} i}^{3}+\gamma\left(\mathrm{I}_{1 i}+\mathrm{I}_{2 i}+\mathrm{I}_{3 i}\right)-\sigma \mathrm{R}-\mu_{i} \mathrm{R}+c_{i-1} \mathrm{R}_{i-1}-c_{i} \mathrm{R}_{i}
\end{aligned}
$$

In writing these equations we assume that the total population is constant in time. Even though the annual mean growth rate in Argentina was $9 \cdot 6 \%$ in the 2000-2005 period, this is a reasonable approximation considering we 
are not interested in describing the dynamics of the model during long time intervals. For a similar model Hethcote has considered a variation of the total population with time for propagation of pertussis in USA throughout 100 years. His results show, that even for this long time lapse, patterns of incidences and age distributions are not considerably affected by considering a constant population size [15]. The rates $\sigma, \sigma_{0}, \tau, \tau^{\prime}$ and $\gamma$ are constant in time and have been defined in the Methods (Models) section, $\mu_{i}$ is the mortality rate for age group $i$, and $c_{i}$ is the rate at which individuals are transferred from an age group to the next because of ageing. We take $c_{i}=1 /\left(a_{i+1}-a_{i}\right)$ for $i=0, \ldots, n_{\mathrm{A}}-2$, in that way, for class $\mathrm{I}_{1}$ for example, in an interval $\Delta t$ a fraction $\Delta t /\left(a_{i}-a_{i-1}\right)$ of individuals in age group $i-1$ are transferred to group $i$ because of ageing, while a fraction $\Delta t /\left(a_{i+1}-a_{i}\right)$ leaves group $i$ to enter $i+1$. Note that there is no younger group than $i=0$ or older than $n_{\mathrm{A}}-1$, so we take $c_{-1}=c_{n_{\mathrm{A}}-1}=0$ in order to cancel the corresponding terms in the set of differential equations. Vaccination is taken into account by transferring individuals from the $\mathrm{S}$ or $\mathrm{P}_{\mathrm{AI}}$ classes to the following increased immunity class when a vaccine dose is applied. This is introduced in the model through the function $\operatorname{vacc}_{i}$, the fraction of individuals at age $a_{i}$ that are successfully immunized. For example, if a dose is applied at age $a_{i}$, the rate $c_{i}$, at which individuals are transferred from $\mathrm{S}_{i-1}$ to $\mathrm{S}_{i}$ because of ageing [first equation in (A1)], has to be multiplied by a factor $\left(1-\operatorname{vacc}_{i}\right)$ because the immunized individuals have been added to $\mathrm{P}_{\mathrm{AI} i}^{1}$ in the last term of the second equation. So, $\operatorname{vacc}_{i}$ is different from 0 only when $a_{i}$ coincides with the age of one of the application doses, $d_{k}$,

$\operatorname{vacc}_{i}=\sum_{k=1}^{n_{\mathrm{d}}} f_{k} \delta_{i k}^{*}, \quad f_{k}=p_{k} . \mathrm{VE}, \quad \delta_{i k}^{*}= \begin{cases}1 & \text { if } a_{i}=d_{k} \\ 0 & \text { if not }\end{cases}$

where $f_{k}$ is the fraction of people at age $d_{k}$ successfully immunized, $p_{k}$ is the coverage of $k$ dose, $n_{\mathrm{d}}$ is the number of doses applied, and VE the vaccine efficacy. In order that this scheme works, ages $a_{i}$ have to be chosen in such a way that each $d_{k}$ coincides with some $a_{i}$.

The last term in the first equation of the system (A1) represents the birth rate. The symbol $\delta$ is the Kroenecker delta, so, $\delta_{i 0}$ indicates that the term is present only for $i=0$. In principle, we assume that every individual is born susceptible, so $B$ is the per capita birth rate and is included in the equation for $\mathrm{S}_{\mathbf{0}}$. In order to keep the total population constant, $B$ should equal the per capita mortality rate, so we take

$B=\sum_{i=0}^{n_{\mathrm{A}}-1} \mu_{i} N_{i}$

where $N_{i}$ is the fraction of the population in age group $i$.

The FOI is the rate at which a susceptible (or partially immune) individual in age group ' $i$ ' acquires infection, and it is calculated as follows:

$\lambda_{i}=\sum_{j} \mathcal{N}_{\mathrm{C}}(i, j)\left(\mathrm{I}_{j}^{*} / N_{j}\right)=\sum_{j} \beta_{i j} \mathrm{I}_{j}^{*} ; \quad \mathrm{I}_{j}^{*}=\mathrm{I}_{1 j}+\rho_{1} \mathrm{I}_{2 j}+\rho_{2} \mathrm{I}_{3 j}$,

where $\mathcal{N}_{\mathrm{c}}(i, j)$ is the number of infective contacts per unit of time between an individual of age group $i$ with individuals of age group $j$, and $\left(\mathrm{I}_{j}^{*} / N_{j}\right)$ represents the effective probability that an individual in age group ' $j$ ' is infective. For 'infective contact' we mean a contact such that if an individual in group $j$ is fully infected $\left(\mathrm{I}_{1}\right)$ and an individual in group $i$ is partially or completely susceptible, then an individual in group $i$ will acquire infection. The contact parameter matrix, $\beta_{i j}=\mathcal{N}_{\mathrm{c}}(i, j) / N_{j}$, gives a measure of the contact rate between individuals of different age groups that is independent of age group sizes.

Once a choice of all the parameters is made, an initial epidemiological state for the population is chosen, and the system of equations (A1) is solved numerically by Euler integration taking a small enough time interval, $\Delta t$, until the stationary state is reached.

\section{Modifications introduced to the model in order to account for the effect of MIT in newborn protection}

We add to our model an extra class $\mathrm{X}_{\mathbf{0}}$. This class includes individuals who acquired maternal antibodies and because of that if they became infected, they would present mild symptomatology $\left(\mathrm{I}_{2}\right)$. Since it is known that the duration of maternal antibodies does not exceed 2 months, the fraction of individuals in $\mathrm{X}_{\mathbf{0}}$ that are not 
vaccinated with the corresponding 2-month dose would go directly to the susceptible class. In contrast, the $\mathrm{X}_{0}$ fraction that gets vaccinated would move to class $\mathrm{P}_{\mathrm{AI}}^{1}$. At this point it is important to note that the new class $\mathrm{X}_{0}$ included in the model is formed only by the $0-2$ months age group. Assuming that mother's antibodies will last $\tau_{\mathrm{A}}$ since they entered class $\mathrm{R}$ and that the typical time that the individuals spend in class $\mathrm{R}$ is $1 / \sigma$, the fraction of mothers that are able to transmit antibodies to their babies is $f_{\mathrm{A}}=\sigma \cdot \tau_{\mathrm{A}} \mathrm{R}_{\mathrm{M}}$. Where $\mathrm{R}_{\mathrm{M}}$ is the fraction of mothers in class $\mathrm{R}$ (fraction of individuals aged between 20 and 35 years that are in class $\mathrm{R}$ ). With these assumptions, $f_{\mathrm{A}}$ represents the fraction of newborns that go to class $\mathrm{X}_{\mathbf{0}}$, and $1-f_{\mathrm{A}}$ represents the fraction that goes to class $\mathrm{S}$. The time evolution of the fraction of individuals in class $\mathrm{X}_{0}$ is given by the equation:

$\frac{\mathrm{d} \mathrm{X}_{0}}{\mathrm{~d} t}=-\lambda_{0} \mathrm{X}_{0}-\mu_{0} \mathrm{X}_{0}-c_{0} \mathrm{X}_{0}+B f_{\mathrm{A}}$

All other equations of the model remain the same as in (A1), with the exception of those given below:

$$
\begin{aligned}
& \left(\frac{\mathrm{dS}_{0}}{\mathrm{~d} t}\right)_{\mathrm{MIT}}=\left(\frac{\mathrm{dS}_{0}}{\mathrm{~d} t}\right)_{\mathrm{A} 1}-B f_{\mathrm{A}}, \\
& \left(\frac{\mathrm{dI}_{20}}{\mathrm{~d} t}\right)_{\mathrm{MIT}}=\left(\frac{\mathrm{dI}_{20}}{\mathrm{~d} t}\right)_{\mathrm{A} 1}+\lambda_{0} \mathrm{X}_{0}, \\
& \left(\frac{\mathrm{dS}_{1}}{\mathrm{~d} t}\right)_{\mathrm{MIT}}=\left(\frac{\mathrm{dS}}{\mathrm{d} t}\right)_{\mathrm{A} 1}+c_{0}\left(1-\operatorname{vacc}_{1}\right) \mathrm{X}_{0}, \\
& \left(\frac{\mathrm{dP}_{\mathrm{AI1}}^{1}}{\mathrm{~d} t}\right)_{\mathrm{MIT}}=\left(\frac{\mathrm{dP}_{\mathrm{AI} 1}^{1}}{\mathrm{~d} t}\right)_{\mathrm{A} 1}+c_{0} \operatorname{vacc}_{1} \mathrm{X}_{0} .
\end{aligned}
$$

where the subindex MIT denotes the magnitude considering maternal immunity transmission and the subindex A1 denotes the expression given in the formula (A1). Taking $\tau_{\mathrm{M}}=2.5$ years we obtain for the $\mathrm{CP}_{1 \mathrm{~A}}$-MDI scenario: $f_{\mathrm{A}}=0 \cdot 12$.

\section{Modifications introduced to the model in order to account for the effect of immune memory on pertussis transmission}

In order to consider the more efficient response exhibited by individuals that have been previously immunized (via infection or three vaccine doses), the classes $\mathrm{P}_{\mathrm{AI}}^{1}$ and $\mathrm{P}_{\mathrm{AI}}^{2}$ were subdivided into two subclasses, one containing individuals that come from $\mathrm{S}$ class (with naive cells) and other containing those that come from $\mathrm{P}_{\mathrm{AI}}^{3}$ class (with immune memory cells). The fraction of individuals in classes $\mathrm{P}_{\mathrm{AI}}^{1}$ and $\mathrm{P}_{\mathrm{AI}}^{2}$ and age group $i$ is written:

$$
\begin{aligned}
& \mathrm{P}_{\mathrm{AI} i}^{1}=\mathrm{P}_{\mathrm{AI} i}^{1(n)}+\mathbf{P}_{\mathrm{AI} i}^{1(m)}, \\
& \mathrm{P}_{\mathrm{AI} i}^{2}=\mathbf{P}_{\mathrm{AI} i}^{2(n)}+\mathbf{P}_{\mathrm{AI} i}^{2(m)},
\end{aligned}
$$

where we have explicitly separated the contributions from individuals with naive cells $(n)$ and immune memory cells $(m)$. Both these subclasses then evolve in time in an independent way with the following difference: when individuals in an $n$ subclass receive a vaccine dose, they increase their immunity gradually following the dynamics of Figure $2\left(\mathrm{P}_{\mathrm{AI}}^{1} \rightarrow \mathrm{P}_{\mathrm{AI}}^{2} \rightarrow \mathrm{P}_{\mathrm{AI}}^{3}\right)$, while individuals in an $m$ subclass go directly to class $\mathrm{C}_{\mathrm{AI}}$ when vaccinated. The dynamics are described by the following equations:

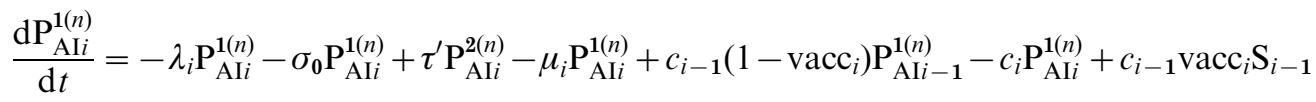

$$
\begin{aligned}
& \frac{\mathrm{dP}_{\mathrm{AI} i}^{1(m)}}{\mathrm{d} t}=-\lambda_{i} \mathbf{P}_{\mathrm{AI} i}^{1(m)}-\sigma_{0} \mathbf{P}_{\mathrm{AI} i}^{1(m)}+\tau^{\prime} \mathbf{P}_{\mathrm{AI} i}^{2(m)}-\mu_{i} \mathbf{P}_{\mathrm{AI} i}^{1(m)}+c_{i-1}\left(1-\operatorname{vacc}_{i}\right) \mathbf{P}_{\mathrm{AI} i-1}^{1(m)}-c_{i} \mathbf{P}_{\mathrm{AI} i}^{1(m)} \\
& \frac{\mathrm{dP}_{\mathrm{AI} i}^{2(n)}}{\mathrm{d} t}=-\lambda_{i} \mathrm{P}_{\mathrm{Al} i}^{2(n)}-\tau^{\prime} \mathrm{P}_{\mathrm{Al} i}^{2(n)}-\mu_{i} \mathrm{P}_{\mathrm{Al} i}^{2(n)}+c_{i-1}\left(1-\operatorname{vacc}_{i}\right) \mathrm{P}_{\mathrm{Al} i-1}^{2(n)}-c_{i} \mathrm{P}_{\mathrm{Al} i}^{2(n)}+c_{i-1} \operatorname{vacc}_{i} \mathrm{P}_{\mathrm{Al} i-1}^{1(n)} \\
& \frac{\mathrm{dP}_{\mathrm{AI} i}^{2(m)}}{\mathrm{d} t}=-\lambda_{i} \mathrm{P}_{\mathrm{AI} i}^{2(m)}-\tau^{\prime} \mathrm{P}_{\mathrm{AI} i}^{2(m)}+\tau^{\prime} \mathrm{P}_{\mathrm{AI} i}^{3}-\mu_{i} \mathrm{P}_{\mathrm{AI} i}^{2(m)}+c_{i-1}\left(1-\mathrm{vacc}_{i}\right) \mathrm{P}_{\mathrm{AI} i-1}^{2(m)}-c_{i} \mathrm{P}_{\mathrm{AI} i}^{2(m)}
\end{aligned}
$$




$$
\begin{aligned}
& \frac{\mathrm{dP}_{\mathrm{AI} i}^{3}}{\mathrm{~d} t}=-\lambda_{i} \mathrm{P}_{\mathrm{AI} i}^{3}-\tau^{\prime} \mathrm{P}_{\mathrm{AI} i}^{3}+\tau \mathrm{C}_{\mathrm{AI} i}-\mu_{i} \mathrm{P}_{\mathrm{AI} i}^{3}+\sigma R+c_{i-1}\left(1-\operatorname{vacc}_{i}\right) \mathrm{P}_{\mathrm{AI} i-1}^{3}-c_{i} \mathrm{P}_{\mathrm{AI} i}^{3}+c_{i-1} \operatorname{vacc}_{i} \mathrm{P}_{\mathrm{AI} i-1}^{2(n)} \\
& \frac{d \mathrm{C}_{\mathrm{AI} i}}{\mathrm{~d} t}=-\tau \mathrm{C}_{\mathrm{AI} i}-\mu_{i} \mathrm{C}_{\mathrm{AI} i}+c_{i-1} \mathrm{C}_{\mathrm{AI} i-1}-c_{i} \mathrm{C}_{\mathrm{AI} i}+c_{i-1} \operatorname{vacc}_{i} \mathrm{P}_{\mathrm{AI} i-1}^{3}+c_{i-1} \operatorname{vacc}_{i} \mathrm{P}_{\mathrm{AI} i-1}^{2(m)}+c_{i-1} \operatorname{vacc}_{i} \mathrm{P}_{\mathrm{AI} i-1}^{1(m)}
\end{aligned}
$$

where the equations for the time derivatives of $\mathrm{S}_{i}, \mathrm{I}_{1 i}, \mathrm{I}_{2 i}, I_{3 i}$ and $\mathrm{R}_{i}$ are the same as in (A1).

\section{Age at first infection}

We estimate the average age at first infection, $A_{1 \mathrm{st}}$, through the expression

$$
A_{1 \mathrm{st}}=\sum_{i} A_{i} \lambda_{i} \mathrm{~S}_{i} / \sum_{i} \lambda_{i} \mathrm{~S}_{i}
$$

where $A_{i}=\left(a_{i+1}+a_{i}\right) / 2$ and the sums are limited to include individuals with ages lower than a given $A_{\max }$ in order to avoid returning to the $\mathrm{S}$ class of adults. In our model, immunized adults lose their immunity and are allowed to become susceptible at a very low rate $\sigma_{0}$, but they should not be considered in the evaluation of $A_{1 \mathrm{st}}$. We only compute $A_{1 \mathrm{st}}$ in the pre-vaccine era and observe that it takes a reasonable value when $A_{\max }$ is between ages 10 and 20 years, when most 'first infections' have already occurred.

\section{Type I mortality}

In this approximation it is assumed that everybody dies exactly at an age equal to the life expectancy: $L$ [25]. In the present work all the mortality rates $\mu_{i}$ are 0 with the exception of that corresponding to the oldest group, $i=n_{\mathrm{A}}-1$, which takes the value: $1 /\left(a_{n_{\mathrm{A}}}-a_{n_{\mathrm{A}}-1}\right)$. As the total population is constant, all the $N_{i}$ are also constant: $N_{i}=\left(a_{i+1}-a_{i}\right) / L$, and the birth rate is $B=1 / L . L=a_{n_{\mathrm{A}}}$ (the highest age considered) $=75$ years in this work. 